\title{
Artery of Percheron Ischaemic Stroke: A Classic Presentation of a Rare Case
}

\author{
Miguel Santos, Ana Rodrigues, Ana Albuquerque, Flávia Santos, Adriana Bandeira, Miriam Magalhães, Maria Banza
}

Department of Internal Medicine, Centro Hospitalar de Leiria, Leiria, Portugal

Received: 02/11/2021

Accepted: $16 / 11 / 2021$

Published: 03/12/2021

\begin{abstract}
How to cite this article: Santos M, Rodrigues A; Santos F, Bandeira A, Magalhães, Banza M. Artery of Percheron ischaemic stroke: a classi presentation of a
\end{abstract} rare case. EJCRIM 2021;8: doi:10.12890/2021_003045.

Conflicts of Interests: The authors declare there are no competing interests.

This article is licensed under a Commons Attribution Non-Commercial 4.0 License

\section{ABSTRACT}

The artery of Percheron (AoP) is a rare anatomic variant, where the paramedian thalami and the rostral midbrain are supplied by an artery emerging from the P1 segment of the posterior cerebral artery. Ischaemic infarction of the artery of Percheron occurs rarely, accounting for 0.1 to $2 \%$ of all ischaemic strokes. AoP occlusion can lead to an infarction of the paramedian thalami and mesencephalon, resulting in a triad of clinical features; namely, altered mental status, vertical gaze palsy and memory impairment. A larger mesencephalon infarction can also feature oculomotor disturbances. We describe here the case of an 88-year-old patient, presenting with this triad of features.

\section{LEARNING POINTS}

- Neurological events such as ischaemic strokes can have a wide clinical presentation.

- Artery of Percheron infarction is characterized by memory impairment, vertical gaze palsy and altered mental status.

- Pattern recognition is fundamental for early diagnosis and optimal care.

\section{KEYWORDS}

Ischaemic stroke, artery of Percheron, memory loss, vertical gaze palsy, altered mental status

\section{INTRODUCTION}

The artery of Percheron (AoP) is a rare anatomic variant, present in 4 to $12 \%$ of the population ${ }^{[1,2]}$. In these cases the paramedian thalami and the rostral midbrain are supplied by an artery emerging from the P1 segment of the posterior cerebral artery ${ }^{[1]}$.

Ischaemic infarction of the artery of Percheron is a rare event, accounting for 0.1 to $2 \%$ of all ischaemic strokes ${ }^{[1]}$. Occlusion of the AoP may lead to an infarction of the paramedian thalami and mesencephalon, clinically featuring a triad of altered mental status, vertical gaze palsy and memory impairment ${ }^{[2,3]}$. A larger mesencephalon infarction can also feature oculomotor disturbances ${ }^{[2]}$.

We describe the case of an88-year-old patient, presenting with all these clinical features.

\section{CASE DESCRIPTION}

We describe the case of an 88-year-old male patient, fully independent in his daily activities (scoring 0 points on the Modified Rankin Scale). He had a clinical history of hypertension and paroxystic atrial fibrillation. He was not on any anticoagulant medication.

The patient was admitted to the Emergency Department1hour after a sudden loss of consciousness. This episode lasted for about $2 \mathrm{minutes}$, according to an eyewitness. It started suddenly and resolved spontaneously. There were no involuntary movements or loss of sphincter control, according to this witness. At admission, the patient was conscious but confused. He would answer incorrectly when asked about 
his location, but could identify correctly the present day. He was able to perform simple tasks on command, except for opening his eyes. He was haemodynamically stable, with a blood pressure of $126 / 78 \mathrm{mmHg}$ and a heart rate of $55 \mathrm{bpm}$. The neurological examination revealed he could not open his eyes spontaneously or on command. His pupils were isocoric and isoreactive but he could not perform ocular rotation with his left eye. Muscular strength and body sensitivity were tested and had no alterations. He presented neither aphasia nor dysarthria when speaking.

At admission he was submitted to a head CT scan, which was normal, Angio-CT had no evidence of major vessel occlusion or endoluminal clots. Blood work, including inflammatory markers and myocardial necrosis markers, was unremarkable. An ECG showed no relevant abnormalities (sinus rhythm with normal QRS complex and no ST segment alteration).

Twenty-four hours after admission, the patient remained confused, maintaining the same findings on neurological examination. Furthermore, it was noted that his confusion was in part due to the fact that he had also a short-term memory loss, not identified at the time of admission. Another head CT scan was performed at this time, showing thalamic nuclei and left paramedian mesencephalic location hypodensity, suggesting a recent ischaemic event in the artery of Percheron territory.

The patient was admitted to the Internal Medicine ward for clinical surveillance, aetiological investigation and rehabilitation. During this phase head MRI was carried out for better characterization of the lesions described previously (Figs. 1-3).
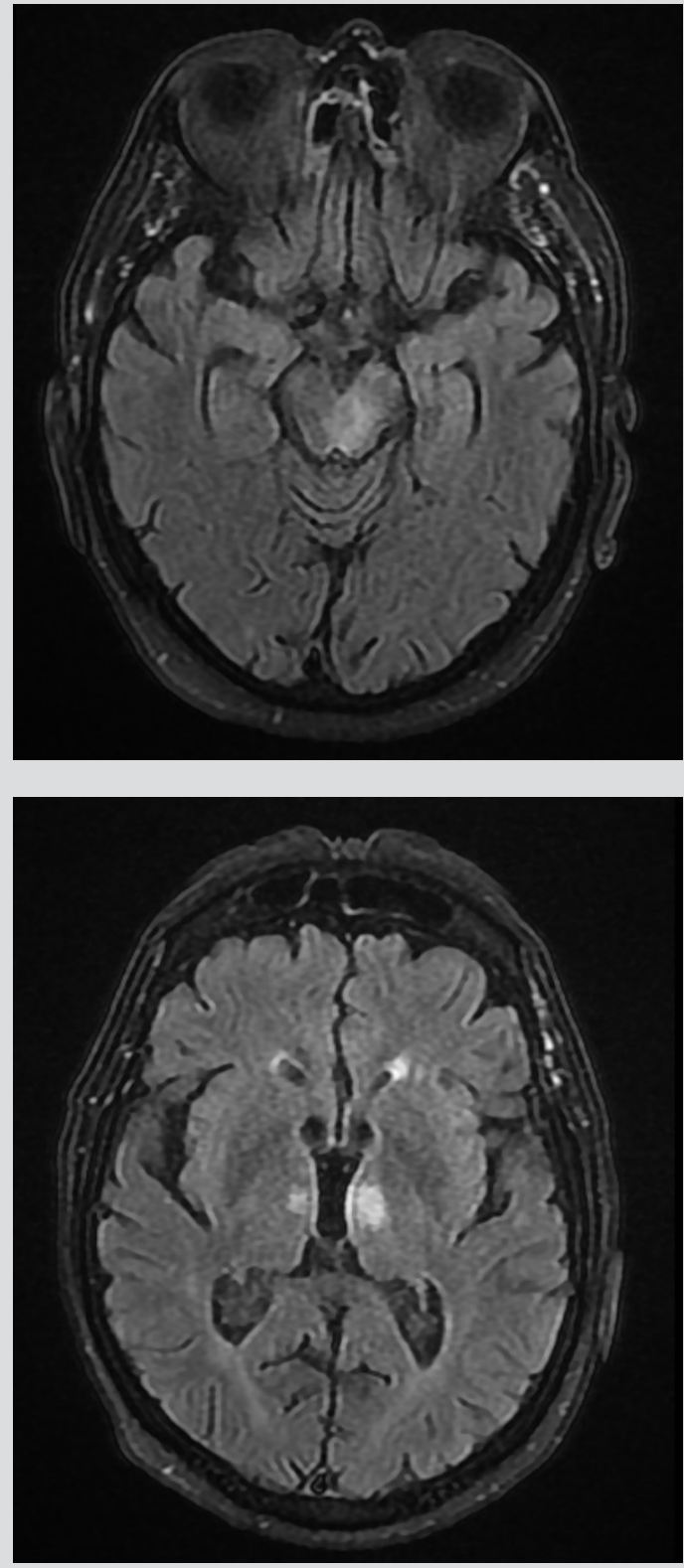

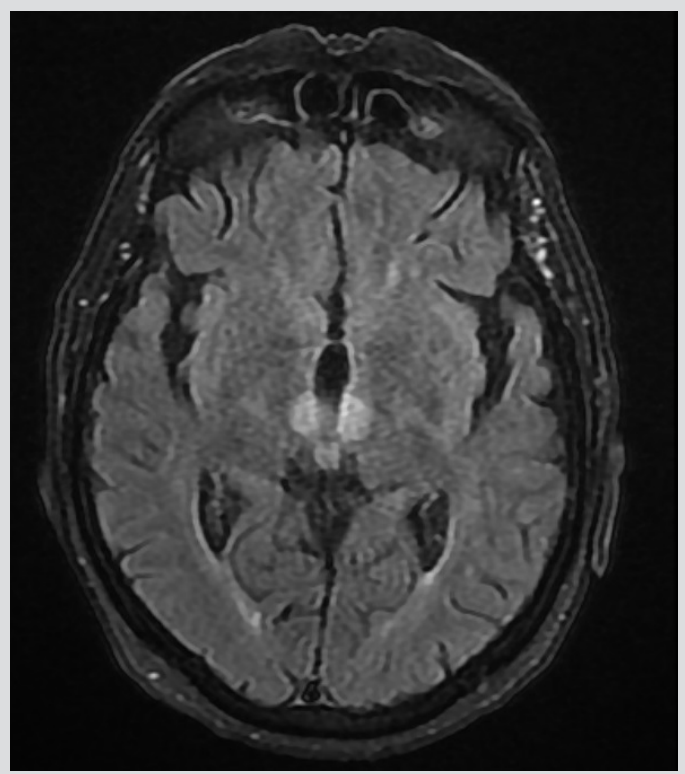

Figure 1. Head MRI demonstrating left mesencephalic lesion

Figure 2. Head MRI demonstrating bithalamic lesions

Figure 3. Head MRI demonstrating bithalamic lesions 


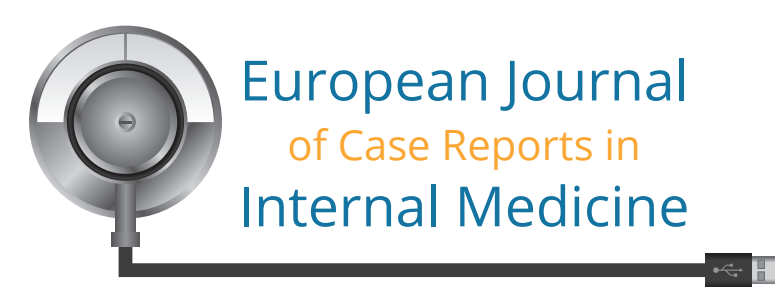

During the following week, the patient underwent physical rehabilitation, improving his ability to open the right eye. Although he maintained some memory lapses, he could now identify correctly where he was. He maintained inability to open his left eye and perform rotation of this eye.

\section{DISCUSSION}

This clinical case shows the typical presentation of a very rare event, the ischaemic stroke of the artery of Percheron. This patient presented at admission the characteristic triad of this territory infarction. Since he presented in the emergency room in such an early phase, there were no imaging findings. Unfortunately, as the attending physicians were not familiar with this rare clinical entity, they did not recognize these clinical symptoms and findings as a possible presentation of an acute ischaemic event. Therefore, the diagnosis was delayed by 24 hours, changing the prognosis, as it was by then too late for fibrinolytic therapy.

We wish to highlight the importance of the recognition of this clinical pattern, rare as it may be, as it could mean an early diagnosis and a better outcome for future patients presenting in this manner.

\section{REFERENCES}

1. Kichloo A, Jamal SM, Zain EA, Wani F, Vipparala N. Artery of Percheron infarction: a short review. J Investig Med High Impact Case Rep 2019;7:2324709619867355.

2. Lazzaro NA, Wright B, Castillo M, Fischbein NJ, Glastonbury CM, Hildenbrand PG, et al. Artery of Percheron infarction: imaging patterns and clinical spectrum. Am J Neuroradiol 2010;31(7):1283-1289.

3. Sasi S, Ahmed A, Yousuf W, Vattoth S. Artery of Percheron infarct: arare presentation of acute ischemic stroke in a high-risk antiphospholipid syndrome patient. Case Rep Acute Med 2020;3:46-52. 